21. Frumin H, O'Donnell L, Kerin NZ, Levine F, Nathan LE Jr, Klein SP. Two-dimensional echo- cardiographic detection and diagnostic features of tricuspid papillary fibroelastoma. J Am Coll Cardiol. 1983;2:1016-8.

22. Sun JP, Asher CR, Yang XS, Cheng GG, Scalia GM, Massed AG, et al. Clinical and echocardiographic characteristics of papillary fibroelastomas: a retrospective and prospective study in 162 patients. Circulation. 2001; 103:2687-93.
23. Atalay MK, Taner AT. Gradual enhancement of a large left atrial papillary fibroelastoma on cardiac magnetic resonance: the waiting game. Tex Heart Inst J. 2010; 37:612-3.

24. Burke A, Virmani R. Papillary fibroelastoma: tumors of the heart and great vessels. AFIP Atlas of Tumor Pathology. 1996;16:47-54.

25. Kim KH, Choi JB. Papillary fibroelastoma in tricuspid valve: an unusual cause of atypical chest pain. J Thorac Cardiovasc Surg. 2013;145:1131.

\title{
Surgical ablation of typical atrial flutter refractory to catheter ablation
}

\author{
Fei Lü, MD, PhD, FACC, FHRS, Taibo Chen, MD, Kenneth K. Liao, MD, PhD, FACS, and \\ David G. Benditt, MD, FACC, FRCPC, FHRS, Minneapolis, Minn
}

To our knowledge, surgical dissection of the cavotricuspid isthmus (CTI) for treatment of typical atrial flutter (AFL) has not been reported previously. A surgical approach, however, may be needed for catheter access for AFL ablation in patients with complex congenital structures with or without surgical repair, ${ }^{1}$ or for hybrid approach for atrial fibrillation ablation. ${ }^{2}$

\section{Case Report}

A 35-year-old man with a history of arrhythmogenic right ventricular cardiomyopathy (ARVC) and after implantation of an implantable cardioverter defibrillator (ICD) presented with recurrent symptomatic atrial tachycardias after 2 unsuccessful attempts of prolonged catheter ablation, reportedly for typical AFL and right atrial (RA) tachycardias at other facilities. On presentation to us, a surface electrocardiogram showed typical AFL morphology with a tachycardia cycle length (TCL) of $360 \mathrm{~ms}$ and a 2:1 atrioventricular conduction (Figure 1, top). The echocardiogram showed severely enlarged RA $(97 \mathrm{~mm})$ with mild tricuspid insufficiency and right ventricular (RV) enlargement $(76 \mathrm{~mm})$ with RV ejection fraction of approximately $10 \%$ and left ventricular ejection fraction of $55 \%$ to $60 \%$.

The patient underwent repeat electrophysiological study. An open-irrigation deflectable catheter with a 3.5-mm tip (ThermoCool; Biosense Webster, Inc, Diamond Bar, Calif) was used for mapping and radiofrequency ablation. Entrainment and a 3-dimensional activation map indicated that the

\footnotetext{
From the Cardiac Arrhythmia Center, Division of Cardiovascular Medicine, Department of Medicine, University of Minnesota Medical School, Minneapolis, Minn Disclosures: Authors have nothing to disclose with regard to commercial support. Received for publication Jan 4, 2013; revisions received Feb 19, 2013; accepted for publication March 4, 2013; available ahead of print April 8, 2013.

Address for reprints: Fei Lü, MD, PhD, FACC, FHRS, Cardiac Electrophysiology Laboratories, Department of Cardiovascular Medicine, University of Minnesota, MMC 508, 420 Delaware St SE, Minneapolis, MN 55455 (E-mail: luxxx074@ umn.edu).

J Thorac Cardiovasc Surg 2013;145:e73-5

$0022-5223 / \$ 36.00$

Copyright (C) 2013 by The American Association for Thoracic Surgery http://dx.doi.org/10.1016/j.jtcvs.2013.03.002
}

tachycardia was consistent with typical counterclockwise CTI-dependent AFL. The CTI was mapped carefully and systematically to search for any detectable atrial signals. Only at a more lateral location in the CTI were there detectable residual atrial signals, whereas there were no or minimal atrial signals at other sites along the whole CTI, presumably owing to the previous ablations. Radiofrequency ablation of the CTI at 7:30 o'clock position followed by ablations at 6:30, 5:30, and 5:00 o'clock position in the 50 left anterior oblique projection produced no effects on tachycardia. Subsequently, a very tiny ventricular signal (without detectable atrial signal) could be recorded at a location more medial toward the septum and more distal toward the tricuspid annulus (TA) and the right ventricle in the isthmus. An ablation lesion delivered to this site successfully terminated the tachycardia (Figure 1, bottom). Insurance burns were placed around the nearby area.

Postablation pacing from coronary sinus $5 / 6$ electrodes at a cycle length of $300 \mathrm{~ms}$ re-induced tachycardia with a TCL of $400 \mathrm{~ms}$. After counterclockwise CTI-dependent flutter again was confirmed by a repeat activation map, additional ablation lesions were delivered along the most medial ablation line crossing the previously successful ablation site in the isthmus. One of these ablations in the middle of the CTI along this line again successfully terminated the tachycardia despite the absence of significant atrial signals at either the distal or proximal poles of the ablation catheter. Afterward, there was no inducible tachycardia with or without isoproterenol. The patient was discharged home the following day with warfarin and carvedilol for his ARVC, without apparent complications.

Approximately 1 month after the last ablation attempt, the patient had recurrent symptomatic palpitations associated with atrial tachycardias. Direct current cardioversion was needed twice within 1 month to restore sinus rhythm.

A decision then was made to attempt surgical ablation to interrupt isthmus conduction. The surgical ablation procedure was performed under general anesthesia with endotracheal intubation. A double-lumen endotracheal tube was 

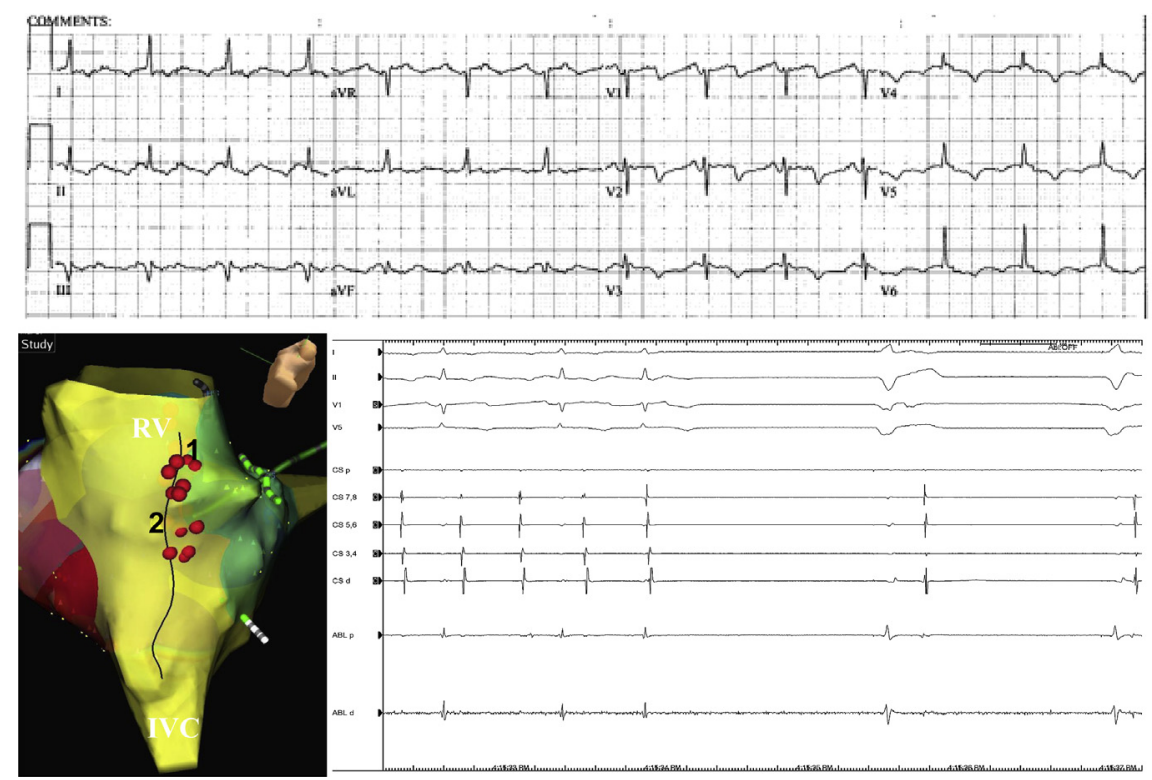

FIGURE 1. Top panel, Atrial tachycardia (cycle length, $360 \mathrm{~ms}$ ) on a 12-lead electrocardiogram. The P-wave morphology during tachycardia is consistent with typical atrial flutter. Bottom panel, Catheter ablation of atrial flutter in the cavotricuspid (CTI). The view of the CTI by 3-dimensional mapping using the EnSite NavX system (St. Jude Medical, St Paul, Minn) is shown at bottom left. Ablation at "1" terminated tachycardia but it remained inducible. Ablation at "2" terminated the induced tachycardia, and it remained noninducible thereafter. Note the pouch at location 2 in the isthmus. Tachycardia was terminated successfully (bottom right) after a 9-second ablation at location " 2 ." Note the absence of atrial electrograms at this successful ablation site. $R V$, Right ventricular; IVC, inferior vena cava. See the text for more details.

used to deflate the right lung. With the right chest elevated $30^{\circ}$, the chest, abdomen, and bilateral proximal lower extremities were prepared with Betadine (Purdue Products, Stamford, Conn) and draped in a sterile fashion. An approximately 4-cm incision was made in the right groin to expose the right femoral artery and vein. After heparinization, a 17F arterial cannula was inserted into the right femoral artery and advanced to the distal abdominal aorta. A multistage $25 \mathrm{~F}$ venous cannula was inserted into the inferior vena cava (IVC). The pleural space was entered through a right minithoracotomy incision made along the right fourth intercostal space in the anterior chest wall. Because of an elevated right diaphragm secondary to a congested liver, retraction sutures had to be placed to bring down the diaphragm to expose the right pericardium. The pericardium was opened longitudinally, approximately 2 inches anterior to the phrenic nerve. The patient then was placed on cardiopulmonary bypass with the heart partially decompressed with $3.5 \mathrm{~L} / \mathrm{min}$ flow established. During the initiation of cardiopulmonary bypass, tachycardia was converted to sinus rhythm at 43 $\mathrm{bpm}$. The right superior vena cava (SVC) then was cannulated with a $30 \mathrm{~F}$ single-stage venous cannula at the RA-SVC junction. Both the SVC and IVC venous drainage cannulae were connected with a Y-tube to the cardiopulmonary bypass machine, resulting in a more decompressed RA. An umbilical tape was placed around the IVC and SVC, respectively, after careful dissection of the pericardial reflection. After tightening these umbilical tapes, the patient was placed on total cardiopulmonary bypass.

A transverse RA incision opening then was made along the right lateral wall and extended toward the medial aspect of the RA toward the TA. The RA wall was noted to be thickened $(5-7 \mathrm{~mm})$ with calcification and scars. After the anatomic locations of the TA and the IVC orifice were identified, an incision (approximately $8-10 \mathrm{~mm}$ in depth and 30 $\mathrm{mm}$ in length) was made transecting the entire CTI from the IVC to the TA (Figure 2). The incision then was closed in 2 layers using a pair of 4-0 Prolene sutures (Ethicon, Inc, Somerville, NJ) in a continuous running fashion. The patient was weaned off cardiopulmonary bypass without difficulties after the RA was closed in a 2-layer fashion. With satisfactory hemostasis, heparin was reversed with protamine; the right lung was ventilated; and both the arterial and venous cannulae were pulled out. The vessel insertion sites were repaired with 5-0 Prolene sutures and the right groin incision was closed with Vicryl sutures (Ethicon, Inc). A $28 \mathrm{~F}$ chest tube was inserted into the right pleural space and a Blake tube (Ethicon, Inc) was inserted into the pericardial space. The intercostal space was closed using 3 separate \#5 Ethibond sutures (Ethicon, Inc). The subcutaneous muscle fascia, subcutaneous tissue, and skin were closed using Vicryl sutures in multiple layers. The patient left the electrophysiology laboratory in a hemodynamically stable condition. 

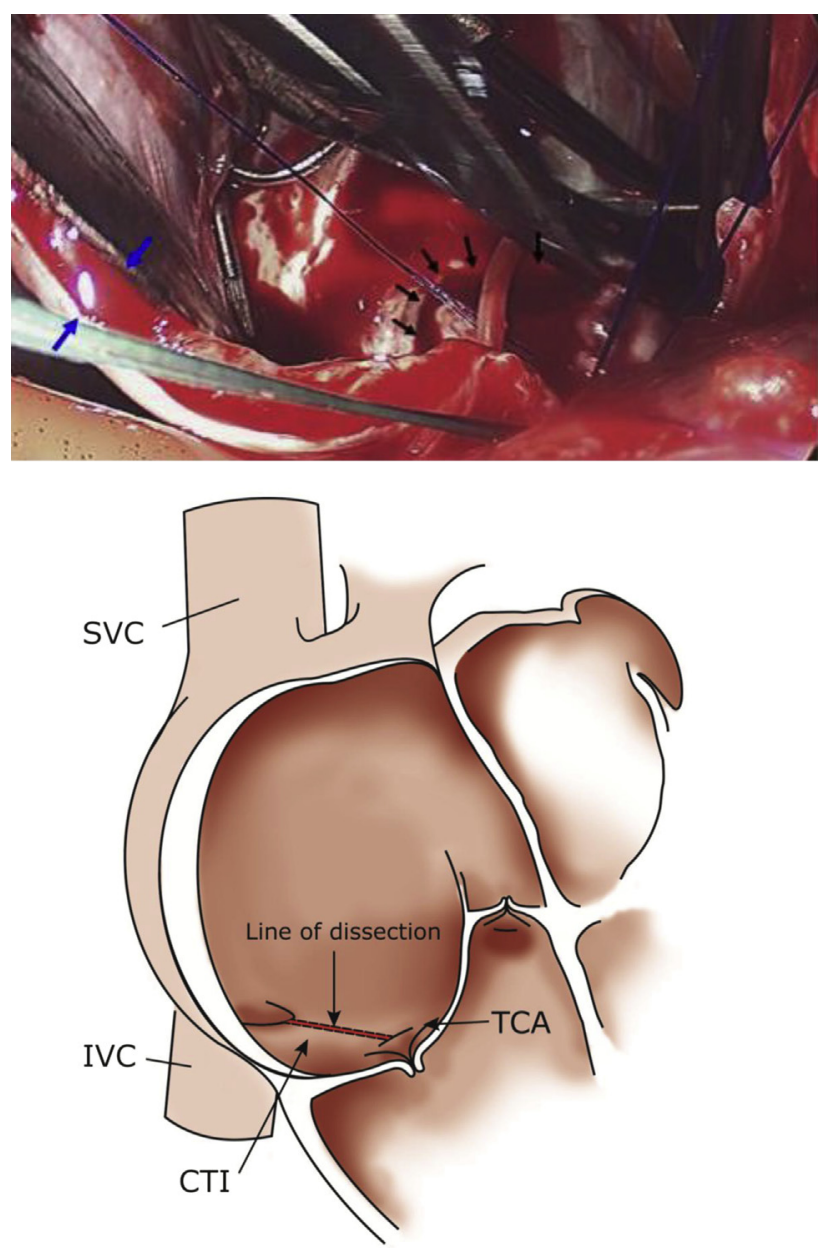

FIGURE 2. Surgical dissection of the cavotricuspid isthmus (CTI). Top, A picture was taken during the surgical procedure. The surgical incision was made from the inferior vena cava (IVC) (left end) to the tricuspid annulus (TCA) (right end). Black arrows indicate the incision and blue arrows indicate the thickened right atrial wall. Bottom, The surgical incision was made in the CTI. SVC, Superior vena cava. See the text for more details.

Without apparent perisurgery complications, the patient was discharged home with carvedilol and warfarin without bridging with subcutaneous heparin. Follow-up evaluation in the clinic showed no apparent complications associated with the surgical ablation procedure and interrogation of his ICD showed no significant atrial tachyarrhythmias over a 6-month period after surgery.

\section{DISCUSSION}

The main finding of this case was the apparent longterm suppression by surgical ablation of CTI-dependent AFL that previously had been refractory to multiple radiofrequency catheter ablation procedures in a patient with ARVC, and marked RA and CTI thickening. Furthermore, our observation in this case suggests that, in the presence of marked CTI thickening, delivery of ablation energy to the CTI at a location without notable atrial electrogram activity does not necessarily preclude possible AFL termination (although recurrence may be expected). Finally, to our knowledge, a CTI-dependent TCL of 400 $\mathrm{ms}$ is likely the longest AFL to have been reported.

Atrial involvement in ARVC is thought to be caused mainly by RV enlargement and tricuspid regurgitation, with a possible contribution by pathologic changes in the atria similar to those in the $\mathrm{RV}^{3}$ A prolonged $\mathrm{P}$-wave duration or abnormal P-wave morphology is seen commonly in patients with ARVC. The incidence of atrial tachyarrhythmias has been reported to be $24 \%$ to $42 \%$ in ARVC patients, ${ }^{4,5}$ and even higher in more advanced disease stage. ${ }^{5}$ Approximately a third of these patients had documented AFL. ${ }^{5}$

The main limitation of this case probably was the lack of mapping during surgical ablation because the tachycardia terminated with atrial manipulation during the initial phase of cardiopulmonary bypass. The transverse RA incision along the RA lateral wall may have altered or destroyed potential arrhythmogenic substrate or circuits in that region, challenging the therapeutic mechanisms of surgical ablation in this case. The evidence of CTI-dependent AFL, confirmed during the several previous catheter ablation procedures, strongly argues in favor of the contribution of an unusually thickened isthmus to this patient's persistent tachyarrhythmia. Nevertheless, follow-up monitoring with ICD clearly indicated the freedom of AFL recurrence after surgical CTI dissection in this case refractory to 3 attempts of aggressive catheter ablation.

\section{References}

1. El Yaman MM, Asirvatham SJ, Kapa S, Barrett RA, Packer DL, Porter CB Methods to access the surgically excluded cavotricuspid isthmus for complete ablation of typical atrial flutter in patients with congenital heart defects. Heart Rhythm. 2009;6:949-56.

2. Muneretto C, Bisleri G, Bontempi L, Curnis A. Durable staged hybrid ablation with thoracoscopic and percutaneous approach for treatment of long-standing atrial fibrillation: a 30-month assessment with continuous monitoring. J Thorac Cardiovasc Surg. 2012;144:1460-5.

3. Basso C, Fox PR, Meurs KM, Towbin JA, Spier AW, Calabrese F, et al. Arrhythmogenic right ventricular cardiomyopathy causing sudden cardiac death in boxer dogs: a new animal model of human disease. Circulation. 2004;109:1180-5.

4. Tonet JL, Castro-Miranda R, Iwa T, Poulain F, Frank R, Fontaine GH. Frequency of supraventricular tachyarrhythmias in arrhythmogenic right ventricular dysplasia. Am J Cardiol. 1991;67:1153.

5. Chu AF, Zado E, Marchlinski FE. Atrial arrhythmias in patients with arrhythmogenic right ventricular cardiomyopathy/dysplasia and ventricular tachycardia. Am J Cardiol. 2010;106:720-2. 